\title{
Luminescence of water in Lake Baikal observed with the Baikal-GVD neutrino telescope
}

A.D. Avrorin ${ }^{1}$, A.V. Avrorin', V.M. Aynutdinov ${ }^{1}$, R. Bannash ${ }^{9}$, I.A. Belolaptikov ${ }^{2}$, V.B. Brudanin $^{2}$, N.M. Budnev ${ }^{3}$, G.V. Domogatsky ${ }^{1}$, A.A. Doroshenko ${ }^{1}, R$. Dvornický ${ }^{2,8, *}$, A.N. Dyachok ${ }^{3}$, Zh.-A.M. Dzhilkibaev ${ }^{1}$, L. Fajt ${ }^{8,9}$, S.V. Fialkovsky ${ }^{5}$, A.R. Gafarov ${ }^{3}$, K.V. Golubkov ${ }^{1}$, N.S. Gorshkov', T.I. Gress ${ }^{3}$, K.G. $\mathrm{Kebkal}^{7}$, O.G. Kebkal ${ }^{7}$, E.V. Khramov ${ }^{2}$, M.M. Kolbin ${ }^{2}$, K.V. Konischev ${ }^{2}$, A.V. Korobchenko ${ }^{2}$, A.P. Koshechkin ${ }^{1}$, V.A. Kozhin ${ }^{4}$, M.V.

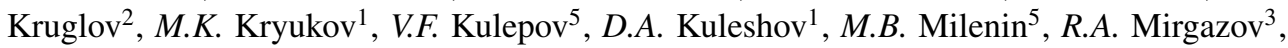
V. Nazari ${ }^{2}$, A.I. Panfilov ${ }^{1}$, D.P. Petukhov ${ }^{1}$, E.N. Pliskovsky ${ }^{2}$, M.I. Rozanov ${ }^{6}$, E.V. Rjabov ${ }^{3}$, V.D. Rushay ${ }^{2}$, G.B. Safronov ${ }^{2}$, B.A. Shaybonov ${ }^{2}$, M.D. Shelepov ${ }^{1}, F$. Šmkovic ${ }^{2,8}, I$. Štekl ${ }^{9}$, A.G. Solovjev ${ }^{2}$, M.N. Sorokovikov ${ }^{2}$, O.V. Suvorova ${ }^{1}$, V.A. Tabolenko ${ }^{3}$, B.A. Tarashansky ${ }^{3}$, S.A. Yakovlev ${ }^{7}$, and A.V. Zagorodnikov ${ }^{3}$

\footnotetext{
${ }^{1}$ Institute for Nuclear Research, Moscow, 117312 Russia

${ }^{2}$ Joint Institute for Nuclear Research, Dubna, 141980 Russia

${ }^{3}$ Irkutsk State University, Irkutsk, 664003 Russia

${ }^{4}$ Institute of Nuclear Physics, Moscow State University, Moscow, 119991 Russia

${ }^{5}$ Nizhni Novgorod State Technical University, Nizhni Novgorod, 603950 Russia

${ }^{6}$ St. Petersburg State Marine Technical University, St. Petersburg, 190008 Russia

${ }^{7}$ EvoLogics, Germany

${ }^{8}$ Comenius University, Mlynská dolina F1, SK-842 48 Bratislava, Slovakia

${ }^{9}$ Czech Technical University in Prague, 12800 Prague, Czech Republic
}

\begin{abstract}
We present data on the luminescence of the Baikal water medium collected with the Baikal-GVD neutrino telescope. This three-dimensional array of light sensors allows the observation of time and spatial variations of the ambient light field. In 2016, we observed a maximum of luminescence activity between July and October.
\end{abstract}

\section{Introduction}

Lake Baikal remains home to various unique species of plants and animals for millions of years, many of which are endemic. The vital conditions for the lake are horizontal and vertical water exchange processes, which supply and distribute the oxygen and organic substances. The study of the hydrodynamic processes in Lake Baikal are of particular interest for earth and life sciences. Beyond limnology, it may improve our understanding of the hydrodynamics of seas and oceans.

The next generation neutrino telescope Baikal-GVD is placed in the southern basin of Lake Baikal about $3.6 \mathrm{~km}$ from shore at a depth of $1366 \mathrm{~m}$. The aim of the experiment is the detection of high energy astrophysical neutrinos, whose sources remain still unknown. In

\footnotetext{
*e-mail: dvornicky@fmph.uniba.sk
} 
particular, the goal is the registration of the Cherenkov radiation emitted when charged particles are passing through the deep water in Lake Baikal. The detector is a three-dimensional array of optical modules (OMs) arranged in independent units called clusters.

In 2016, the demonstration cluster "Dubna" has been upgraded to contain 288 OMs attached on 8 strings, 7 peripheral strings surrounding the central one with a radius of $60 \mathrm{~m}$. Each string carries 36 OMs with $15 \mathrm{~m}$ vertical spacing. The top and the bottom OMs are located at depths of $750 \mathrm{~m}$ and $1275 \mathrm{~m}$, respectively. In the subsequent winter expeditions of 2017 and 2018, two more clusters have been deployed [1].

Apart of Cherenkov radiation, also the ambient background light is registered. The trigger system of each of the three clusters is designed in such a way that signals from each OM in a time window of $5 \mu \mathrm{s}$ are stored, if a trigger condition is fulfilled [2]. In this way, we collect the data on count rates of pulses registered by each particular OM. These are mainly associated with the luminescence of the Baikal water and registered almost continuously. In this article, we present some selected results on luminescence in Lake Baikal.

\section{Optical activity of the Baikal water}

Baikal-GVD is designed to detect the Cherenkov light from charged particles. In open water, light not related to relativistic particles constitutes an unavoidable background to the Cherenkov light. Therefore studies of the related light fields are of crucial importance. The photon flux from the sunlight below a depth of $\sim 700 \mathrm{~m}$ is negligible as shown in previous work [3].

In Fig.1a, the charge distribution of background light is presented. It turns out that the major contribution comes from single photo-electron pulses. In following, we will show that periods of stable optical background noise are intermitted by increased optical activity. We stress that the charge distribution remains unchanged in different periods. Our measurements are performed with a threshold of half a single photo-electron charge. In this way, the dark noise of the photo-multiplier is significantly suppressed.

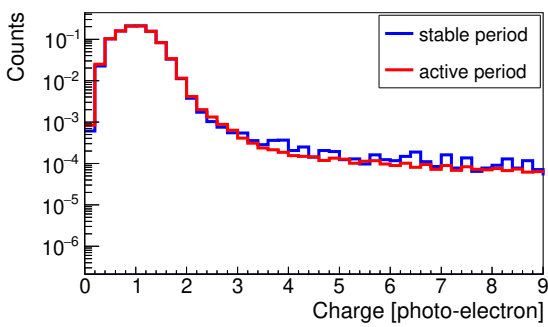

(a)

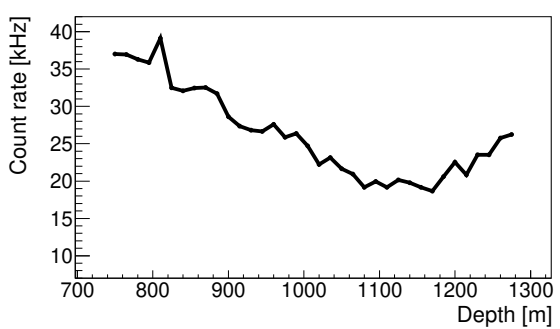

(b)

Figure 1: a) Charge distribution of the registered pulses in units of photo-electrons. b) Count rates as a function of depth, averaging for each depth over the OMs from different strings. The lake bed is at $1366 \mathrm{~m}$ depth. Data from June 2016.

The depth dependence of the ambient light field is the same for all eight strings of a cluster. Averaging over the OMs at the same horizon, we obtain the depth dependence of the background light noise. The average count rates versus the depth are presented in Fig.1b. The analysed data are from June of 2016. This is the period of lowest optical activity (see below), but the form of the depth dependence remains the same for other periods. 


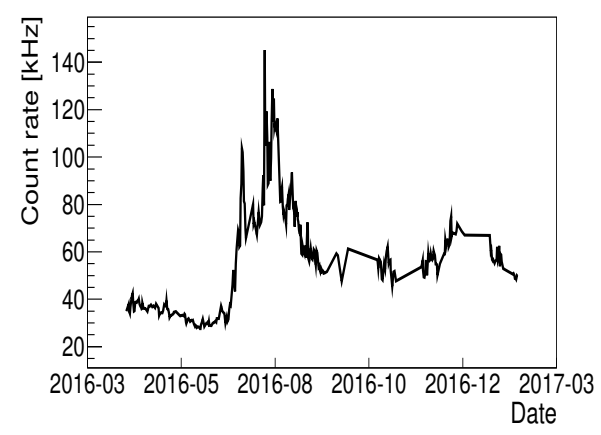

(a)

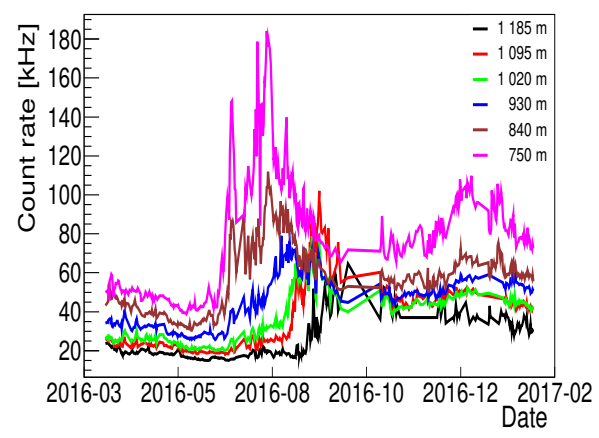

(b)

Figure 2: a) Count rates of a selected OM versus time (April 2016 - February 2017). b) Count rates for the OMs at the same string at different depths. For the sake of simplicity, we show only six of 36 OMs, placed at depths of 750, 840, 930, 1020, 1095, and 1185 meters.

In Fig.2a, we present data on count rates for a selected OM for April 2016 - February 2017. There are two periods of relative stability. In between we clearly see a period of increased optical activity against the stable plateaus. The depth dependence of the effect is displayed in more detail in Fig.2b. The appearance of the outbreak maximum depends on time, starting with the top modules. Indeed, we observe a layer of highly luminescent water moving from the top to the bottom of the lake. By comparing the maximum for different depths, we obtain a velocity profile of the flows. In the beginning of August, the estimated speed reached its maximal value of $\sim 45 \mathrm{~m} /$ day, while it remained almost constant ( $\sim 8 \mathrm{~m} /$ day) till the end of September, i.e. when the activity asymptotically reached the background plateau. On the other hand, we observed a practically constant background noise without a period of high luminescence activity in 2017. The observed pattern is similar to previous investigations with NT200 detector (see [4]).

Due to the currents in Lake Baikal, the string geometry deviates from its vertical direction. To take these deviations into account, an acoustic positioning system for Baikal-GVD has been developed (For more details see the contribution to this conference [5]). Our observations show two periods of extreme deviations of the strings, in September of 2016 and of 2017. Torrent flows in the lake may produce a remarkable tilt of the string from its vertical position, two examples of which are displayed in Figs.3a and $3 \mathrm{~b}$. For the same period, we present the data on count rates in Figs. $3 \mathrm{c}$ and $3 \mathrm{~d}$. We do not find a correlation between the torrent flows of the deep water and the luminescence activity of the lake.

\section{Conclusions}

We have presented data on the luminescence in Lake Baikal which have been collected by the Baikal-GVD neutrino telescope. We found an increase of the luminescence activity intermitting periods of relatively stable optical background. Moreover, we find that the maximum of the optical activity observed in 2016 propagated from top to bottom, with a maximum speed of $45 \mathrm{~m} /$ day. We did not find a correlation between the torrent flows and the increase of the luminescence activity. 


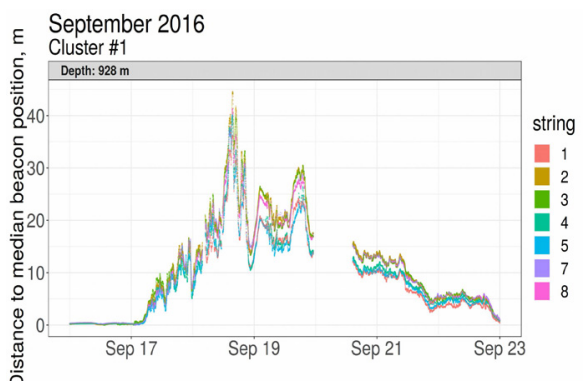

(a)

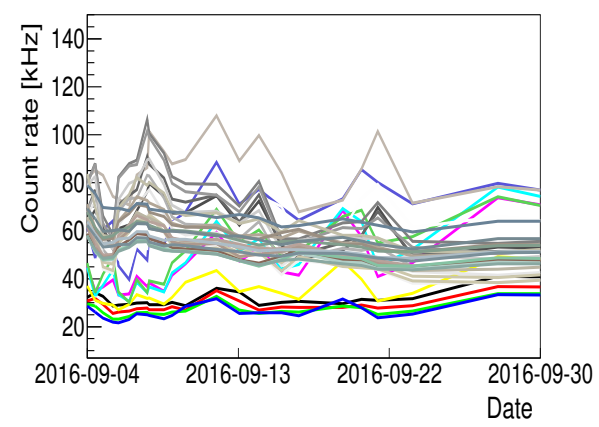

(c)

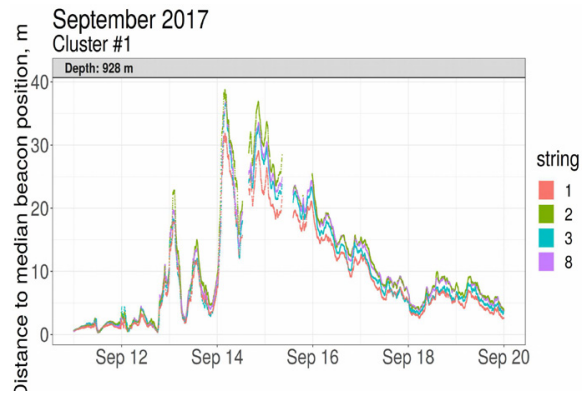

(b)

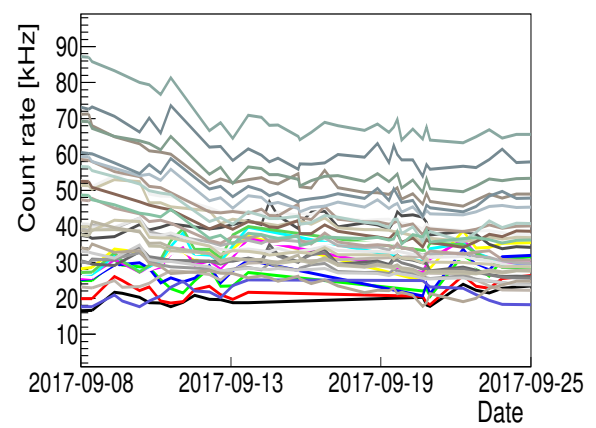

(d)

Figure 3: a) Deviations of the beacons from their median positions at different strings. Shown are data from autumn 2016, when the deviations of the strings from their median positions were extremal. b) Same as for a), with data from 2017. c) Count rates of 36 OMs at the same string. Data shown have been taken in the period, when the deviations of the string from the median position were extremal. d) Same as for c), with data from 2017.

\section{Acknowledgements}

This work was supported by the Russian Foundation for Basic Research (Grants 16-2913032, 17-02-01237).

\section{References}

[1] V.M. Aynutdinov et al., these proceedings

[2] O.V. Suvorova et al., RICAP 2018 conf.proc., in press

[3] Bezrukov et al., Izvestiya, Atmospheric and Oceanic Physics 34, 85 (1998)

[4] Belolaptikov et al., Izvestiya, Atmospheric and Oceanic Physics 34, 78 (1998)

[5] A. Avrorin et al., these proceedings 